\title{
Probabilistic Cluster Signature for Modeling Motion Classes
}

\author{
Shandong Wu, Y.F. Li and Jianwei Zhang
}

\begin{abstract}
In this paper, a novel 3-D motion trajectory signature is introduced to serve as an effective description to the raw trajectory. More importantly, based on the trajectory signature, a probabilistic model-based cluster signature is further developed for modeling a motion class. The cluster signature is a mixture model-based motion description that is useful for motion class perception, recognition and to benefit a generalized robot task representation. The signature modeling process is supported by integrating the EM and IPRA algorithms. The conducted experiments verified the cluster signature's effectiveness.
\end{abstract}

\section{INTRODUCTION}

$\mathrm{M}$ OTION trajectory can play an important role in characterizing diverse kinds of motions of humans, robots and other objects. Ude et al. [1] used motion trajectory to describe human movements in transferring the motions to a humanoid robot. Bennewitz et al. [2] investigated motion trajectory-based human pattern representation for robot learning using hidden Markov model (HMM). Common human actions, behaviors and activities were modeled by motion trajectory in many studies [3], [4] where the recognition of gestures, gaits, etc., was validated. In view of the articulated structure of humans or robots, multiple concurrent motion trajectories can be extracted by tracking the body parts of interest such as the head, hands, feet, etc. [5], to characterize complicated full body motions.

However, in the literature, a motion trajectory was normally used directly in its raw data form, which is inflexible to use as it relies heavily on the absolute positions. In fact, it is suitable to refer to the concept of shape descriptor [6] to build effective motion trajectory descriptions, which can outperform the raw trajectory data in offering generalized motion representation.

In the existing work, some shape descriptors have been developed and used. Simple contour functions such as chain code, centroid-contour distance and R-S curve just admit ordinary performance [6]. The descriptors based on Fourier descriptor (FD) [7], wavelet coefficient [8] and curvature

This work was supported by grants from the Research Grants Council of Hong Kong [Project No. CityU117106 and CityU117507].

Shandong Wu is with the Computer Vision Lab of School of Electrical Engineering and Computer Science, University of Central Florida, 4000 Central Florida Blvd., Orlando, FL 32816, USA (e-mail: sdwu@cs.ucf.edu).

Y.F. $\mathrm{Li}$ is with the Department of Manufacturing Engineering and Engineering Management, City University of Hong Kong, 83 Tat Chee Avenue, Kowloon, Hong Kong (e-mail: meyfli@cityu.edu.hk).

Jianwei Zhang is with the Department of Informatics, University of Hamburg, Vogt-Kölln-Str. 30, D-22527, Hamburg, Germany (e-mail: zhang@informatik.uni-hamburg.de). scale space images [9] can represent shape in a coarse-to-fine manner, in which just partial salient features are of concern for shape description. This explains why they are actually unable to represent shapes uniquely. In particular, small-scale time-dependent features of motions cannot be recovered from Fourier transform based representation. The algebraic curve and moment functions [10] suffer from occlusion as they make use of global features. The mathematical descriptors such as NURBS [11] and B-spline [12] need a fitting process that inevitably causes inaccuracy in shape representation. In particular, the B-spline based description may result in recognition ambiguity as it is hard to compare B-spline parameters directly for recognition because a piece of curve is not uniquely described by a single set of control points [12].

In this paper, a novel 3-D motion trajectory signature descriptor is firstly introduced, and then a model-based cluster signature is investigated using the probabilistic modeling methods for establishing effective descriptions for a motion class/pattern. While the motion trajectory signature is a description to an individual trajectory, the cluster signature corresponds to a motion pattern, which is characterized by a set of trajectory signatures of a cluster of similar trajectory instances from the same motion class.

\section{Motion TRAJECTORY SignATURE}

For a free-from 3-D motion trajectory parameterized by $\Gamma(t)=\{X(t), Y(t), Z(t) \mid t \in[1, \mathrm{~N}]\} \quad$, where $\mathrm{N}$ is trajectory length (frame number) and $t$ is the temporal stamp of trajectory sampling, the following motion trajectory signature has been proposed based on Euclidean curvature $(\mathcal{K})$, torsion $(\tau)$ and their first order derivatives with respect to the Euclidean arc-length parameter $S$ [13], [14],

$$
S=\left\{\left[\kappa(t), \kappa_{s}(t), \tau(t), \tau_{s}(t)\right] \mid t \in[1, \mathrm{~N}]\right\}
$$

where $\kappa_{s}=d \kappa / d s, \tau_{s}=d \tau / d s$.

The above signature is a complete description to the entire raw trajectory data. This is the same with the first-level signature defined in our previous work in [15]. As the signature is based on the local features, it admits the computational locality. Hence, rich descriptive invariants can be deduced from the signature [14].

An arbitrary length of regular (i.e. for all $t, \dot{\Gamma}(t) \neq 0$ ) motion trajectory can be described by a single signature. For the irregular trajectories, the stationary points are firstly detected by examining $\dot{\Gamma}(t)=0$ and then they are removed to regulate the trajectory to be a regular one to calculate the signature. This interprets why the signature is able to describe most motion trajectories extracted from a complicated motion. 
Taking the trajectory $\Omega$ shown in Fig. 1 as an example, its trajectory signature is illustrated in Fig. 2 with the four signature profiles of $\kappa, \tau, \kappa_{s}$ and $\tau_{s}$.

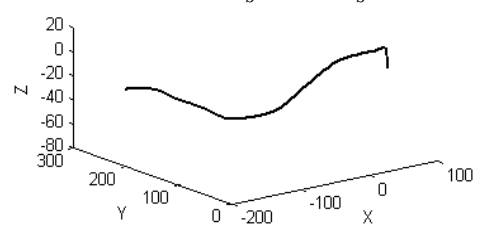

Fig. 1. A piece of 3-D motion trajectory $\Omega$.
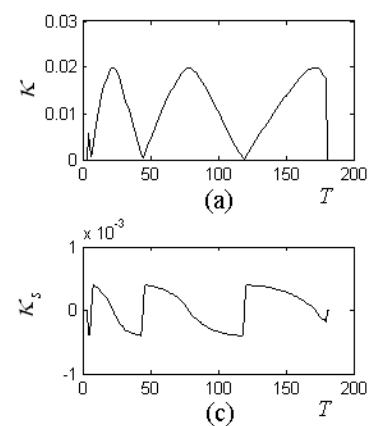

(c)

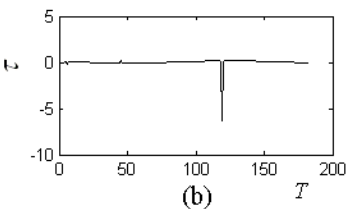

(b)

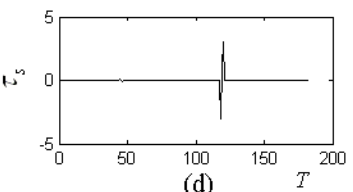

(d)
Fig. 2. The signature (implemented by the approximate signature [13]) of

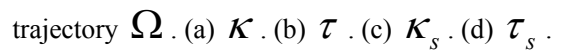

\section{Model-Based Cluster Signature}

\section{A. Cluster Signature Modeling}

A motion class is essentially a common motion pattern representing a meaningful motion type. Hence, not limited to building the signature for describing a single motion trajectory, the signature description for a motion class is of more importance to serve as a generalized motion description. Usually a motion class is characterized by a cluster of signatures of similar trajectory instances. A so-called cluster signature here is proposed for describing a motion classes. It is a probabilistic model-based representation learned from the density estimation to a cluster of motion trajectory signatures. Inheriting the rich invariants of the trajectory signature, the cluster signature is more effective than the common model-based methods that are based on the raw motion data.

Assume that the number of motion classes is $N$, then via training, $N$ models will be learned that are characterized by respective model parameters $\left\{\Theta_{i}\right\}_{i=1}^{N}$. Assume that $X_{i}$ contains $M$ signature samples (note that the samples do not necessitate the same length), i.e. $X_{i}=\left\{X_{i, m}\right\}_{m=1}^{M}$, which serve as the training sample to train an individual model $\Theta_{i}$, these samples are firstly re-arranged in the form of $X_{i}=\left[X_{i, 1} X_{i, 2} \cdots X_{i, m} \cdots X_{i, M}\right]$. Then the underlying probability density function (PDF) of $X_{i}$ can be estimated by a Gaussian mixture model (GMM),

$$
P\left(X_{i} \mid \Theta_{i}\right)=\sum_{k=1}^{K} w_{k} N\left(X_{i} ; \mu_{k}, \Sigma_{k}\right)
$$

where $K$ is the number of mixing Gaussian components, $\left\{w_{k}\right\}$ is the set of mixing weights $w_{k}=P\left(k \mid \Theta_{i}\right)$ meeting $\sum_{k=1}^{K} w_{k}=1$ and $N\left(X_{i} ; \mu_{k}, \Sigma_{k}\right)$ denotes the multivariate Gaussian function (mean $\mu$, covariance $\Sigma$, and data dimension $d$ ),

$f_{(\mu, \Sigma)}\left(X_{i}\right)=\frac{1}{\sqrt{2 \pi}^{d} \sqrt{\operatorname{det} \sum}} \exp \left(-\frac{1}{2}\left(X_{i}-\mu\right)^{T} \Sigma^{-1}\left(X_{i}-\mu\right)\right)$

It is worthy emphasizing the format of the signature $X_{i, m}$ for the GMM modeling. The temporal index $T$ of a motion trajectory is incorporated into the signature data to form an augmented signature in the format of $X_{i, m}=\left[T(t) ; k(t) ; k_{s}(t) ; \tau(t) ; \tau_{s}(t)\right]$. The reason of incorporating $T$ is that it is capable of capturing the temporal characteristics of a motion. However, notice that because signatures may have different lengths, $T$ may need a pre-normalization to make the signatures being aligned reasonably in temporal correspondence. Assume the lengths of the $M$ signatures are $\left\{T_{i}\right\}_{i=1}^{M}$, a bound of temporal range is defined by $T_{\text {low }}$ and $T_{u p}$ (usually $T_{\text {low }}=1$ and $\left.T_{u p}=\max _{i \in[1, M]}\left\{T_{i}\right\}\right)$, then the temporal indexes of each signatures are normalized to the range of $\left[T_{\text {low }}, T_{u p}\right]$. Note that this pre-normalization just adjusts the temporal indexes for the purpose of reasonable temporal alignments among signatures, in which the real signature data are not affected. In this manner, the dimension $d$ of $f_{(\mu, \Sigma)}\left(X_{i}\right)$ will be 5 (1-D temporal index plus 4-D signature components). It is also pointed out that for keeping consistent alignment between temporal stamp and signature data, the pre-normalization may be further improved by the pair-wise normalization using different scaling constants between different pairs of critical points along the trajectories.

From the definition of a GMM model, a motion class $\Theta_{i}$ is characterized by the corresponding model parameter set $\Theta_{i}=\left\{w_{k}, \mu_{k}, \Sigma_{k}\right\}_{k=1}^{K}$. The key problem now is the model parameter estimation. Assume that the number of classes $N$ is known a priori and the training samples are labeled knowing which sample belongs to which class, the expectation-maximization (EM) algorithm [16] and the iterative pairwise replacement algorithm (IPRA) [17] are combined to learn an optimal GMM parameter set $\Theta_{i}$.

The EM algorithm is an iterative maximum likelihood estimation (MLE) algorithm. It seeks to maximize the likelihood function by gradient descent technique. Here, the likelihood function is defined by the log-likelihood 


$$
L\left(\Theta_{i}\right)=\sum_{m=1}^{M} \log \sum_{k=1}^{K} w_{k} N\left(X_{i, m} ; \mu_{k}, \Sigma_{k}\right) \text {. The GMM }
$$

parameter $\Theta^{*}{ }_{i}$ is learned following the MLE principle, $\Theta_{i}^{*}=\arg \max L\left(\Theta_{i}\right)$.

Yet, it should be noted that the results of the EM algorithm depend much on the initial parameter values because the EM algorithm is always monotonically convergent to find the local maximum. Therefore the choice of the initial parameter values is critical. In practice, the GMM mixing number $K$ should be estimated at first, followed by the initialization of respective GMM component parameters $\left\{w_{k}, \mu_{k}, \Sigma_{k}\right\}_{k=1}^{K}$. Thus, guessing a good initial value to $K$ is the most important basis for successful model learning. Usually, $K$ is just fixed from user's guess or determined by trial-and-error [15]. Relying on user's intervention is inflexible, and the actual situation is that a user has to invest a lot of efforts to guess at a good value to $K$ for each GMM model. To improve on this and achieve automatic model learning, it is proposed to use the IPRA algorithm to refine the results of the EM algorithm to obtain optimal learning results. That is, this solution integrates the EM and IPRA algorithms.

The basic principle is as follows: firstly, the EM algorithm is activated by an arbitrary initialization of the mixing number $K$; secondly, the IPRA algorithms refines (merges) the resulting GMM model parameter $\Theta_{i}$, in which $K$ is also adjusted correspondingly.

Initially, $K$ is set to a relatively big number to guarantee that the $K$-component GMM model is adequately capable of modeling the density. As a bigger $K$ leads to more accurate modeling, the more complex the signatures are, the bigger the $K$ should be set initially by users. For example, set $K=50$, if it is estimated that a 50 -component GMM is sufficient to describe the signatures. In fact, it is algorithmic safe to set $K$ an arbitrary big number. Once $K$ is initialized, the k-means method is used to estimate the initial GMM parameter values $\Theta_{i}{ }^{(0)}=\left\{w_{k}{ }^{(0)}, \mu_{k}{ }^{(0)}, \Sigma_{k}{ }^{(0)}\right\}_{k=1}^{K}$. With these initial parameter estimations, the EM algorithm iterates the E (expectation) step and the M (maximization) step [15], [16], until the convergence condition arrives. Here the convergence condition is defined as a threshold about the function $L\left(\Theta_{i}\right)$, i.e. $L^{(t+1)}\left(\Theta_{i}\right) / L^{(t)}\left(\Theta_{i}\right)-1<C_{\text {stop }}$.

Because $K$ was initialized by an arbitrary, big number, the convergence of the EM process does not guarantee that the resulting GMM model must be an optimal resolution. For instance, certain components may be very close or similar. Thus, the IPRA algorithm is immediately applied to further refine the results of the EM algorithm. The core principle of the IPRA is, among the resulting mixture components $\left\{w_{k}, \mu_{k}, \Sigma_{k}\right\}_{k=1}^{K}$ from the EM algorithm, to construct a minimum spanning tree connecting all the components, and then the similarities of all mixing-components are measured and examined to iteratively merge the most similar pairs of the mixing components, along which the component parameters are updated correspondingly. After arriving at the predefined minimum similarity threshold, the IPRA process stops, eventually giving rise to the optimized GMM model parameters (the mixing number $K$ and the corresponding component parameters $\left.\left\{w_{k}, \mu_{k}, \Sigma_{k}\right\}_{k=1}^{K}\right)$.

For two mixing components represented by $\left[w_{1}, N_{1}\right]$ and $\left[w_{2}, N_{2}\right]$ respectively, the similarity measurement between them is defined based on the Hellinger metric as follows,

$$
H\left(\left[w_{1}, N_{1}\right],\left[w_{2}, N_{2}\right]\right)=\left|\sqrt{w_{1} w_{2}}\left(1-2 \int \sqrt{N_{1} N_{2}} d x\right)\right|
$$

where, denoting the signature data dimension by $d$,

$$
\int \sqrt{N_{1} N_{2}}=(2 \sqrt{2 \pi})^{d}\left|\Sigma_{1}\right|^{1 / 4}\left|\Sigma_{2}\right|^{1 / 4} N\left(0, \mu_{1}-\mu_{2}, 2 \Sigma_{1}+2 \Sigma_{2}\right)
$$

If $H\left(\left[w_{1}, N_{1}\right],\left[w_{2}, N_{2}\right]\right)$ is smaller than the predefined merging similarity threshold $H_{\text {merge }}$, then $\left[w_{1}, N_{1}\right]$ and $\left[w_{2}, N_{2}\right]$ will be merged, and the Method-of-Moments algorithm is used to update the component parameters,

$$
\begin{gathered}
w=w_{1}+w_{2} \\
\mu=\frac{w_{1}}{w} \mu_{1}+\frac{w_{2}}{w} \mu_{2} \\
\Sigma=\frac{w_{1}}{w} \Sigma_{1}+\frac{w_{2}}{w} \Sigma_{2}+\frac{w_{1} w_{2}}{w^{2}}\left(\mu_{1}-\mu_{2}\right)\left(\mu_{1}-\mu_{2}\right)^{T}
\end{gathered}
$$

As above stated, applying the EM together with the IPRA algorithms, all the optimal model parameters $\left\{\Theta_{i}\right\}_{i=1}^{N}$ representing respective motion classes can be obtained. The model parameter set $\Theta_{i}=\left\{w_{k}, \mu_{k}, \Sigma_{k}\right\}_{k=1}^{K}$ is defined as the cluster signature, which in essence admits an abstract description to a motion class. It should be noted that the cluster signature defined here is different from the signature modeling in [15]. The work in [15] deals with the length-fixed second-level signature which was simply modeled by the GMM using the EM algorithm solely.

It is emphasized that the proposed solution of integrating the EM and IPRA algorithms can automate the process of the model-based learning. Initializing $K$ with an arbitrary number of big enough can reliably lead to a fully automatic learning procedure. This is more efficient than solely using the EM algorithm. The computational time for the cluster signature learning depends on both the size of training samples and the initialization value of $K$.

\section{B. Instantiating a Generalized Trajectory Signature}

Based on a learned cluster signature for a motion class, a generalized trajectory signature instance can be instantiated from the GMM model. This can predict a trajectory signature from the cluster signature according to a given predictor, and it can also provide an intuitive interface for perceiving the 
characteristics of a motion class. Motion perception is useful for enhancing human-robot interactions.

The signature instantiation is resolved using the Gaussian mixture regression (GMR) method [17]. The regression problem is to reconstruct a general signal form from a set of observations. Arranging the variables in an observation with two sub-sets in the form of $O=\{X, Y\}$, the observation is divided to a predictor variable set represented by the $p$-dimensional $X$ and a response variable set represented by the $q$-dimensional $Y$. If the joint density of the observation $O$ falls in the GMM, then when partitioning the joint density by $f_{X, Y}=f_{Y \mid X} f_{X}$, both $f_{Y \mid X}$ and $f_{X}$ also have the GMM distribution. This is the basis to derive the GMR aiming at estimating the conditional expectation of $Y$ given $X$. For the signature-based regression, the observations are a cluster of trajectory signature instances of a motion class, and it has been known that the signature cluster does fall in the GMM. For an augmented signature $O_{a}=\{T, S\}$ where $S$ represents the signature data and $T$ denotes the temporal index, the GMR is followed to get a generalized trajectory signature by estimating the conditional expectation value of $S$ given $T$.

Using the method in Section III.A, a cluster signature is firstly obtained by modeling a cluster of trajectory signature samples $O_{a}=\{T, S\}$ by the GMM distribution,

$$
f_{T, S}(t, s)=\sum_{k=1}^{K} w_{k} N\left(t, s ; \mu_{k}, \Sigma_{k}\right)
$$

For each Gaussian component $N\left(T, S ; \mu_{k}, \Sigma_{k}\right)$, re-arrange the parameter items $\mu_{k}$ and $\Sigma_{k}$ in terms of the predictor $T$ and response $S$ as follows,

$$
\mu_{k}=\left[\begin{array}{l}
\mu_{k T} \\
\mu_{k S}
\end{array}\right], \Sigma_{k}=\left[\begin{array}{ll}
\Sigma_{k T T} & \Sigma_{k T S} \\
\Sigma_{k S T} & \Sigma_{k S S}
\end{array}\right]
$$

Then each Gaussian component is partitioned and the GMM density function can be transformed into the following form,

$$
f_{T, S}(t, s)=\sum_{k=1}^{K} w_{k} N\left(s \mid t ; \hat{\mu}_{k}(t), \hat{\Sigma}_{k}\right) N\left(t ; \mu_{k T}, \Sigma_{k T T}\right)
$$

where

$$
\begin{gathered}
\hat{\mu}_{k}(t)=\mu_{k S}+\Sigma_{k S T} \Sigma_{k T T}{ }^{-1}\left(t-\mu_{k T}\right) \\
\hat{\Sigma}_{k}=\Sigma_{k S S}-\Sigma_{k S T} \Sigma_{k T T}{ }^{-1} \Sigma_{k T S}
\end{gathered}
$$

The conditional PDF of $S \mid T$ can be formulated by

$$
f_{S \mid T}(s \mid t)=\sum_{k=1}^{K} C_{k}(t) N\left(s ; \hat{\mu}_{k}(t), \hat{\Sigma}_{k}\right)
$$

where the mixing weight $C_{k}$ is calculated as follows, based on the marginal density of $T$,

$$
C_{k}(t)=\frac{w_{k} N\left(t ; \mu_{k T}, \Sigma_{k T T}\right)}{\sum_{k=1}^{K} w_{k} N\left(t ; \mu_{k T}, \Sigma_{k T T}\right)}
$$

Thus, given $T$, the conditional expectation and covariance of $S$ can be estimated as follows,

$$
\begin{aligned}
& \hat{\mu}(t)=E(S \mid T=t)=\sum_{k=1}^{K} C_{k}(t) \hat{\mu}_{k}(t) \\
& \hat{\Sigma}(t)=\operatorname{Cov}(S \mid T=t)=\sum_{k=1}^{K} C_{k}(t)^{2} \hat{\Sigma}_{k}
\end{aligned}
$$

That is, given a predictor vector $\hat{T}$ (a set of temporal indexes), via evaluating $\hat{\mu}(t)$ at each temporal index $t \in \hat{T}$, a generalized trajectory signature $\hat{S}=\{\hat{\mu}(t) \mid t \in \hat{T}\}$ can be produced. At the same time, the covariance matrix $\hat{\Sigma}(t)$ indicates the generalization extent around $\hat{\mu}(t)$ at each point $t$. It is worthwhile to point out that in the GMR, $\hat{T}$ can differ from $T$ in length and the temporal interval, and the resulting signature $\hat{S}$ will have the same length with $\hat{T}$. This offers the feasibility to produce diverse signature instances with different signature lengths and points' distribution by configuring different predictors.

\section{Bayesian Signature Recognition}

A Bayesian signature recognition engine was built before [15] and is used here again. The posterior probability $\log P\left(\Theta_{i} \mid X_{q}\right)$ is used for signature recognition based on the maximum a posterior (MAP) criterion. This means that for $N$ motion classes denoted by $\left\{\Theta_{i}\right\}_{i=1}^{N}$, the query $X_{q}$ is classified into the class $\Theta_{M A P}$ by examining $X_{q}$ as an observation to each GMM model,

$$
\Theta_{M A P}=\underset{\Theta}{\arg \max }\left[\log P\left(\Theta_{i} \mid X_{q}\right)\right]
$$

\section{EXPERIMENTS}

In the experiment, sign language is used to demonstrate the cluster signature-based motion class description. A signed word is performed by a single human hand, and the underlying regular motion trajectory is extracted as the spatiotemporal representation of the word. The raw 3-D motion trajectory is acquired by stereo tracking and is smoothed using the wavelet smoother [13], [14]. Fig. 3 shows the 3-D trajectories extracted from a set of sign words demonstrated by human.

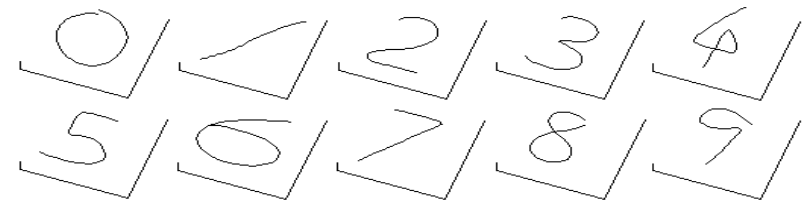

Fig. 3. Motion trajectories of a set of human demonstrated sign words. 


\section{A. Sign Cluster Signature Estimation and Perception}

As shown in Fig. 4, three signatures instances of different lengths of the sign class ' 5 ' are clustered. They have 191, 127 and 153 sampling points, respectively. From Section III.A, their temporal indexes are firstly pre-normalized to the range of $[1,191]$. Then the GMM and IPRA procedures are applied to the three signatures with the initial parameters of mixing Gaussian number $K=30$, the stopping threshold of the EM algorithm $C_{\text {stop }}=1 \mathrm{e}-9$, and the IPRA merging similarity threshold $H_{\text {merge }}=0.001$. Finally an optimal cluster signature (Fig. 5) is modeled by a GMM that actually consists of 20 mixing Gaussian models. Each mixing Gaussian model is represented by an ellipse in which the star symbol indicates the data center and the covered field of the ellipse indicates the varying range of the corresponding partial signature data being modeled. From the visualized cluster signature, the varying range of the motion class can be intuitively perceived from the sequence of ellipses (Gaussian models).
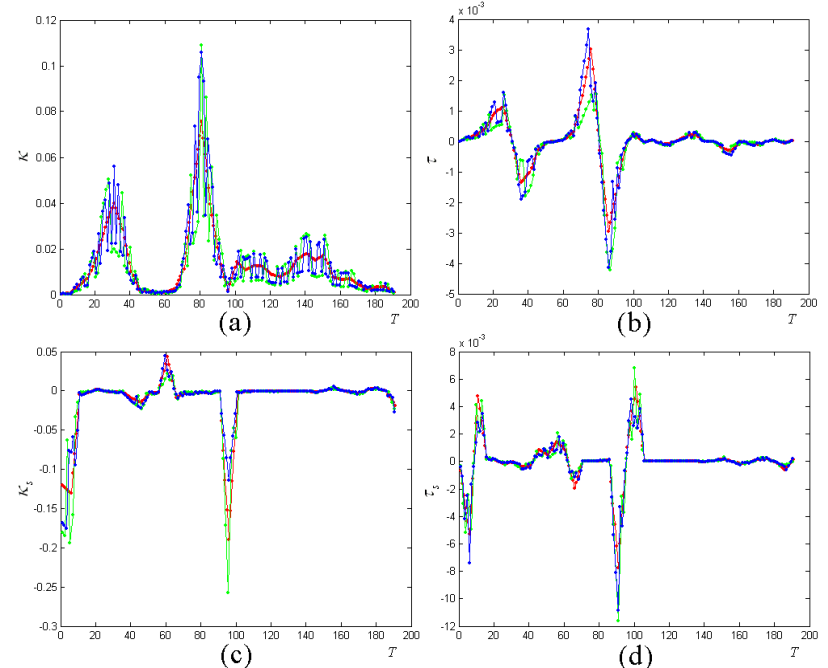

Fig. 4. The three signature instances plotted in red (length 191), green (length

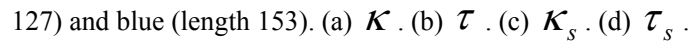
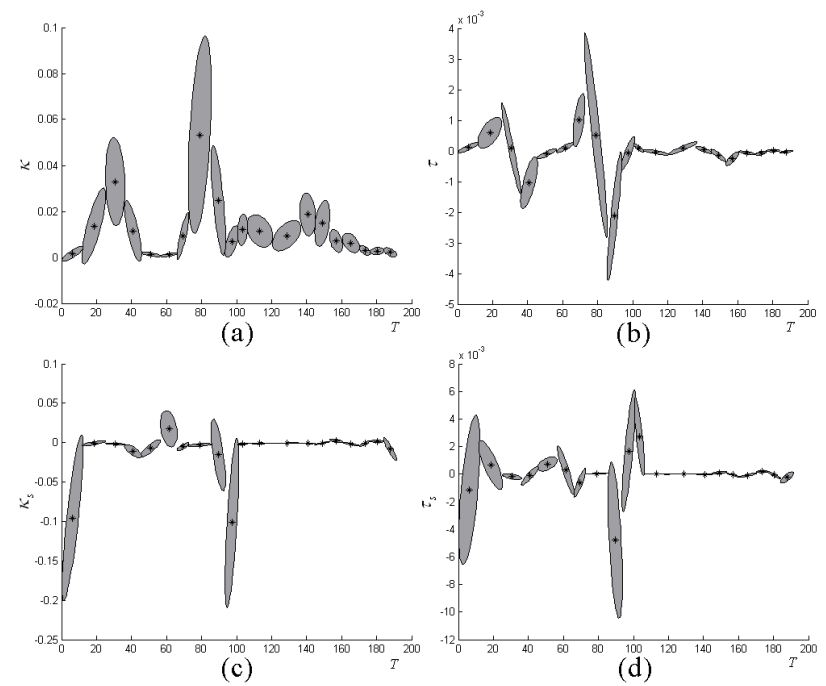

Fig. 5. The GMM-based cluster signature of the signatures instances shown

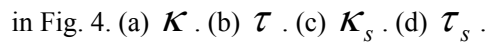

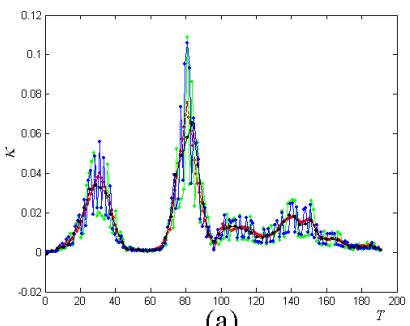

(a)

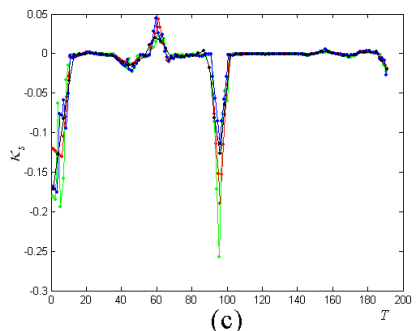

(c)

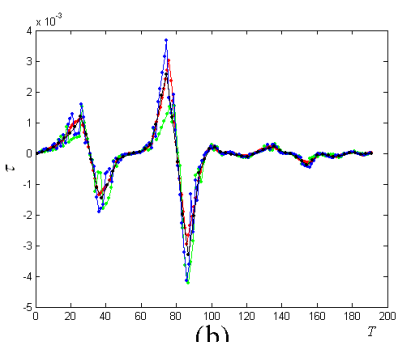

(b)

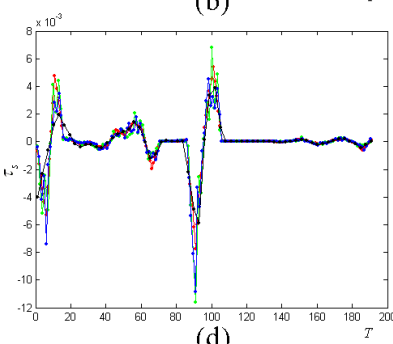

(d)
Fig. 6. Signature generalization (in black) from the cluster signature with

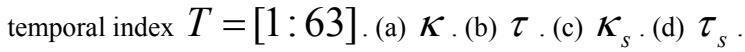

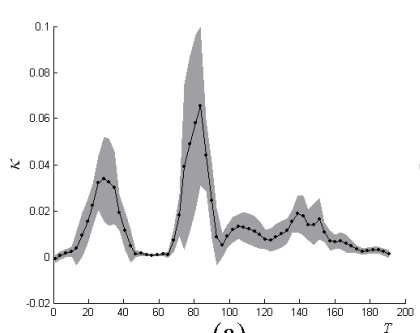

(a)
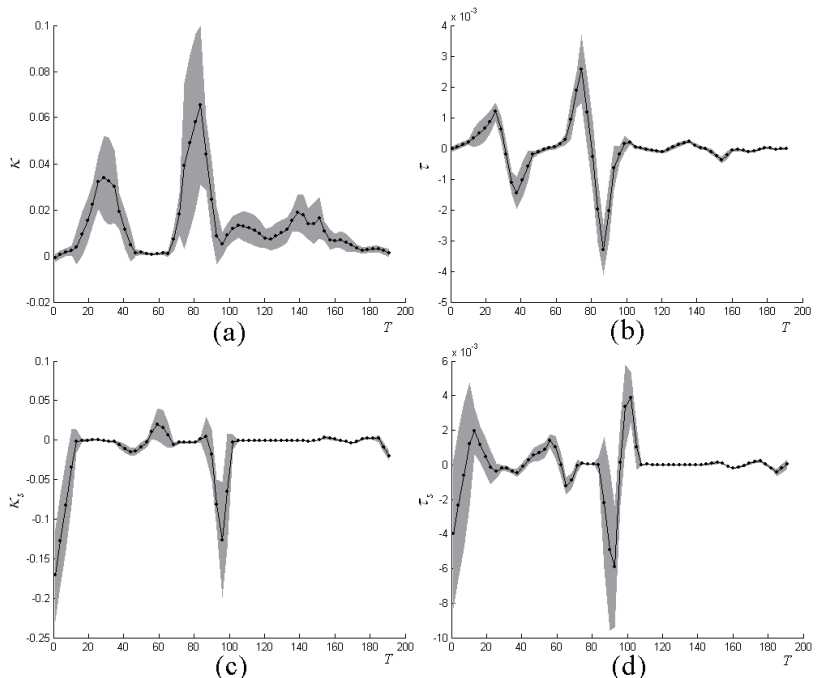

(b)

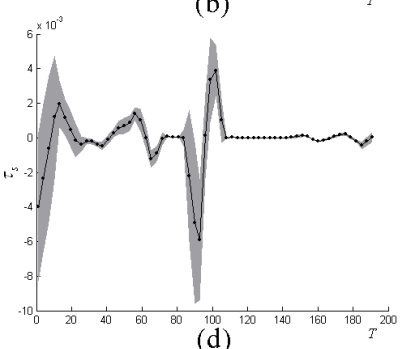

Fig. 7. Varying range (gray fields) of the generalized signature shown in Fig.

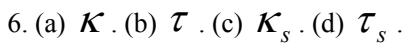

Next, the cluster signatures regression is demonstrated by setting different predictors. Fig. 6 shows a generalized trajectory signature that is produced with the predictor $T=[1: 63]$. The generalized signature is overlaid (in black) in the three signature instances to compare its generalization degree. It is observed that the regressed signatures can serve as good representative signature of the sign word ' 5 '. As said before, this kind of generalized signature can be produced with free configurations of the temporal length and points' distribution to support possibly diverse class descriptions.

In the course of the GMR procedure, the variability of the mean of each element along the generalized signature is restricted to a generalization range that is controlled by the covariance matrix in equation (17). This can be visualized around each temporal index (point). As demonstrated in Fig.7, the gray fields indicate the generalization range of the generalized trajectory signature shown in Fig. 6. This gives an explicit perception to the possible variability of each 
trajectory point, and then the entire generalized signature.

The above results illustrate the visualized form of a cluster signature, from which, the characteristics of a motion class can be intuitively perceived. In the next experiment, the cluster signature-based sign class recognition is demonstrated.

\section{B. Sign Recognition Test}

The Bayesian signature recognition is tested using the UCI KDD high quality Auslan sign trajectory dataset [18] (There are 2,565 samples in total) and its performance is compared with a direct signature matching method in [13]. Referring to [13], the dynamic time warping (DTW) method was applied to recognize a sign motion by the nonlinear matching of the first-level signature. Two instances of the sign word 'hurry' and 'exit' are shown in Fig. 8. The sign trajectories are smoothed by the wavelet smoother using wavelet DB5 and extracting the third level coefficients. Of all the samples of a class, half are used as training samples to learn a cluster signature, and the other half are inputted to do 1-NN recognition. The recognition is repeated more than 50 times on a common PC (Pentium 4 CPU 3.00GHz, 512M RAM) by randomly picking a number of classes and samples, which gives rise to an average recognition performance as recorded in Table 1 and Table 2, respectively. It is observed that the cluster signature behaves better in both accuracy and efficiency. In particular, the improvement on recognition accuracy is due to the utilization of the prior knowledge through model learning. In contrast, The DTW method cannot do that as it just matches two signatures, straightforwardly. More statistical sign recognition results can be found in [19].

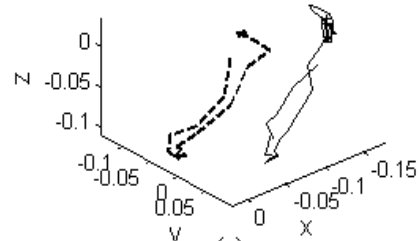

(a)

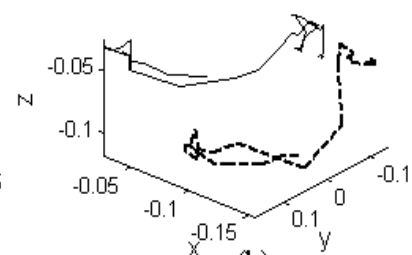

(b)
Fig. 8. Sign samples of word 'hurry' (a) and 'exit' (b).

Table 1. Recognition accuracy comparison.

\begin{tabular}{|c|c|c|c|}
\hline \multirow{2}{*}{ Solutions } & \multicolumn{3}{|c|}{ Number of classes } \\
\cline { 2 - 4 } & 2 & 4 & 8 \\
\hline DTW trajectory signature matching & $91.79 \%$ & $87.05 \%$ & $80.56 \%$ \\
\hline Bayesian cluster signature recognition & $92.73 \%$ & $88.58 \%$ & $83.02 \%$ \\
\hline
\end{tabular}

Table 2. Recognition efficiency comparison (units of milliseconds per query).

\begin{tabular}{|c|c|c|c|}
\hline \multirow{2}{*}{ Solutions } & \multicolumn{3}{|c|}{ Number of classes } \\
\cline { 2 - 4 } & 2 & 4 & 8 \\
\hline DTW trajectory signature matching & 753 & 1287 & 2206 \\
\hline Bayesian cluster signature recognition & 163 & 187 & 191 \\
\hline
\end{tabular}

\section{CONCLUSION}

Based on a 3-D motion trajectory signature, a GMM-based cluster signature is developed in this paper for describing motion patterns. More importantly, we propose a solution that combines the EM and IPRA algorithms for the pursuit of automatic model learning. This proves more efficient than the solely use of the EM algorithm. In addition, the GMR-based signature regression problem is also formulated, from which a generalized trajectory signature can be instantiated from an abstract cluster signature according to a given predictor.

The probabilistic model-based cluster signature can admit wide applications for motion characterization. It is shown that the cluster signature is not only useful for perceiving a motion class by the model visualization, but also exhibits good performance for motion recognition. The cluster signature description can also serve as an invariant and effective robot task representation for supporting generalized robot learning.

\section{REFERENCES}

[1] A. Ude, C.G. Atkeson, and M. Riley, Programming full-body movements for humanoid robots by observation, Robotics and Autonomous Systems, vol. 47, pp. 93-108, 2004.

[2] M. Bennewitz, W. Burgard, G. Cielniak, and S. Thrun, Learning motion patterns of people for compliant robot motion, International Journal of Robotics Research, vol. 24, no. 1, pp. 31-48, 2005.

[3] J. Martin, D. Hall, and J. Crowley, Statistical gesture recognition through modeling of parameter trajectories, Lecture Notes in Computer Science, vol. 1739, pp. 129-140, 1999.

[4] D. Meyer, J. Psl, and H. Niemann, "Gait classification with HMMs for trajectories of body parts extracted by Mixture densities," in Proc. British Machine Vision Conference, England, 1998, pp. 459-468.

[5] J. Min and R. Kasturi, "Extraction and temporal segmentation of multiple motion trajectories in human motion," in Proc. IEEE International Conference on Computer Vision and Pattern Recognition, Washington, D.C., June 2004, pp. 118-122.

[6] V.V. Kindratenko, On using functions to describe the shape, Journal of Mathematical Imaging and Vision, vol. 18, no. 33, pp. 225-245, 2003.

[7] P.R.G. Harding and T.J. Ellis, "Recognizing hand gesture using Fourier descriptors," in Proc. the 17th International Conference on Pattern Recognition, vol. 3, Cambridge, UK, 2004, pp. 286-289.

[8] G. Chuang and C.C. Kuo, Wavelet descriptor of planar curves: theory and applications, IEEE Transactions on Image Processing, vol. 5, no. 1, pp. 56-70, 1996

[9] F. Mokhtarian, S. Abbasi, and J. Kittler, "Robust and efficient shape indexing through curvature scale space," in Proceedings of British Machine Vision Conference, Edinburgh, UK, 1996, pp. 53-62.

[10] M. K. Hu, Visual pattern recognition by moment invariants, IEEE Transactions on Information Theory, vol. 8, no. 2, pp. 179-187, 1962.

[11] J. Aleotti and S. Caselli, "Trajectory clustering and stochastic approximation for robot programming by demonstration," in Proc. IEEE/RSJ International Conference on Intelligent Robots and Systems, Alberta, Canada, August, 2005, pp. 1029- 1034.

[12] F.S. Cohen, Z. Huang, and Z. Yang, Invariant matching and identification of curves using B-splines curve representation, IEEE Transactions on Image Processing, vol. 4, no. 1, pp. 1-10, 1995.

[13] S.D. Wu, Y. F. Li, and J. W. Zhang, "Signature descriptor for free form trajectory modeling," in Proc. IEEE International Conference on Integration Technology, Shenzhen, China, 2007, pp. 167-172.

[14] S.D. Wu and Y.F. Li, On signature invariants for effective motion trajectory recognition, The International Journal of Robotics Research, vol. 27, no. 8, pp. 895-917, Aug. 2008

[15] S.D. Wu, Y.F. Li and J.W. Zhang, "A hierarchical motion trajectory signature descriptor," in Proc. IEEE International Conference on Robotics and Automation, Pasadena, CA, May, 2008, pp. 3070-3075.

[16] J.A. Bilmes, A gentle tutorial of the EM algorithm and its application to parameter estimation for Gaussian Mixture and Hidden Markov Models, International Computer Science Institute, TR-97-021.

[17] H.G. Sung, Gaussian Mixture regression and classification, Ph.D. Dissertation, Rice University, 2004.

[18] UCI KDD Auslan Archive, [Online], Available: http://kdd.ics.uci.edu/databases/auslan2/auslan.html.

[19] S.D. Wu and Y.F. Li, Flexible signature descriptions for adaptive motion trajectory representation, perception and recognition, Pattern Recognition, vol. 42, no. 1, pp. 194-214, Jan. 2009. 